\title{
Evaluación de los recursos turísticos en Canasí.
}

\author{
Evaluation of tourist resources in Canasí.
}

Lic. Adriana Morales Blanco. ${ }^{1}$

\begin{abstract}
The fundamental objective of the present investigation is to evaluate the resources tourist present in Canasí Cove. To carry out the investigation methods like the documental revision they are used, the observation, the method Delphi, interviews to leaders, squares and specialists, and tools like the inventory records and hierarchization are used. This allowed discover its potential for the sustainable development of the area in within short, medium- and long-term time planning. The result of the investigation constitutes to complete and to present the inventoried resources, categorized and hierarchized in an inventory record. It is possible to demonstrate the real value that Canasí possesses for the development of the tourism in the north coast of Santa Cruz.
\end{abstract}

Key Words: Resources; Inventory; Canasí; Tourism.

\section{Resumen}

El objetivo general de la presente investigación es evaluar los recursos presentes en una de las abras más bellas del mundo (Abras de Canasí, provincia de Mayabeque, Cuba) con un enfoque turístico. Para realizar la investigación se utilizan métodos como la revisión documental, la observación, el método Delphi, entrevistas a dirigentes, cuadros y especialistas, y se emplean herramientas como las fichas de inventario y jerarquización. El resultado final de la investigación lo constituye el completamiento y presentación de los recursos y atractivos turísticos inventariados, categorizados y jerarquizados en una ficha de inventario. Esto permitió definir su potencialidad, y las posibles oportunidades que estratégicamente puedan aprovecharse para la realización de acciones que consideren el desarrollo sustentable del área en un marco temporal a corto, mediano y largo plazos. Se logra demostrar el valor real que posee Canasí para el desarrollo del turismo en el litoral norte de Santa Cruz.

Palabras Clave: Recursos; Inventario; Canasí; Turismo.

\footnotetext{
${ }^{1}$ Facultad de Turismo. Universidad de La Habana, La Habana, Cuba, amoralesb00@gmail.com
} 


\section{Introducción}

Durante décadas, el turismo ha experimentado un continuo crecimiento y una profunda diversificación, hasta convertirse en uno de los sectores económicos que crecen con mayor rapidez en el mundo. El turismo mundial guarda una estrecha relación con el desarrollo y se inscriben en él un número creciente de nuevos destinos.

En la Política Económica y Social del Partido y la Revolución para el Turismo en el período del 2016-2021, específicamente en el lineamiento 208 se precisa que es prioridad "continuar incrementando la competitividad de Cuba en los mercados turísticos, diversificando las ofertas, potenciando la capacitación de los recursos humanos y la elevación de la calidad de los servicios con una adecuada relación "calidad-precio". El lineamiento 212, promueve también la necesidad de inversión extranjera: "Continuar incrementando la participación de la industria y los servicios del país en los recursos que se utilizan en la operación e inversión turística. La participación de la industria nacional deberá desarrollarse con financiamiento a largo plazo."

Desde inicios del milenio Abras de Canasí ubicado en el litoral del municipio Santa Cruz del Norte, provincia de Mayabeque, Cuba, ha sido objeto de estudio por geógrafos cubanos, punto de interés de los organismos vinculados al ordenamiento territorial debido a la belleza natural del entorno (Centro Nacional de Áreas Protegidas, 2009) y oportunidad para cumplir con las legislaciones antes mencionadas. De hecho, el Ministerio de Turismo (MINTUR) incluyó en su cartera de oportunidades para la inversión extranjera la propuesta de creación de un parque temático en la zona en el año 2017

Hasta el momento se conocen estudios geológicos de la zona, aunque ninguno evalúa los recursos allí presentes con un enfoque turístico. Por eso la presente investigación se propone evaluar los recursos turísticos de Abras de Canasí, a través de un inventario que bien podría resultar un modelo para estudios de otras propuestas de inversión en productos turísticos con condiciones naturales e infraestructurales similares. Ello permitirá identificar el potencial de la zona y posibles modalidades a desarrollar, facilitará el proceso de planificación física, ordenamiento territorial, y gestión estratégica de la zona de Jibacoa, al cual pertenece el abra, y enriquecerá las propuestas de inversión que se establezcan del lugar.

\section{Procedimiento metodológico para la evaluación de los recursos.}

Luego de una exhaustiva, larga y detallada búsqueda y revisión de bibliográfica en todas las fuentes de información posibles se encuentran varios procedimientos y metodologías para clasificar y evaluar recursos y atractivos turísticos. Entre ellos los de Jean Camara, Gómez Ortega, y Morcate Labrada (2019), MINCETUR (2006), OMT (1978), Sierra 
López (2006), Font Aranda (2013), Gavín Morales, D.E. (2019) y el del Ministerio de Culturas y Turismo (2011).

Finalmente se selecciona como procedimiento metodológico el utilizado por el Ministerio de Culturas y Turismo (2011) y se tomarán elementos de las consideraciones de otros autores para desarrollar cada etapa de aquel (Figura 1)

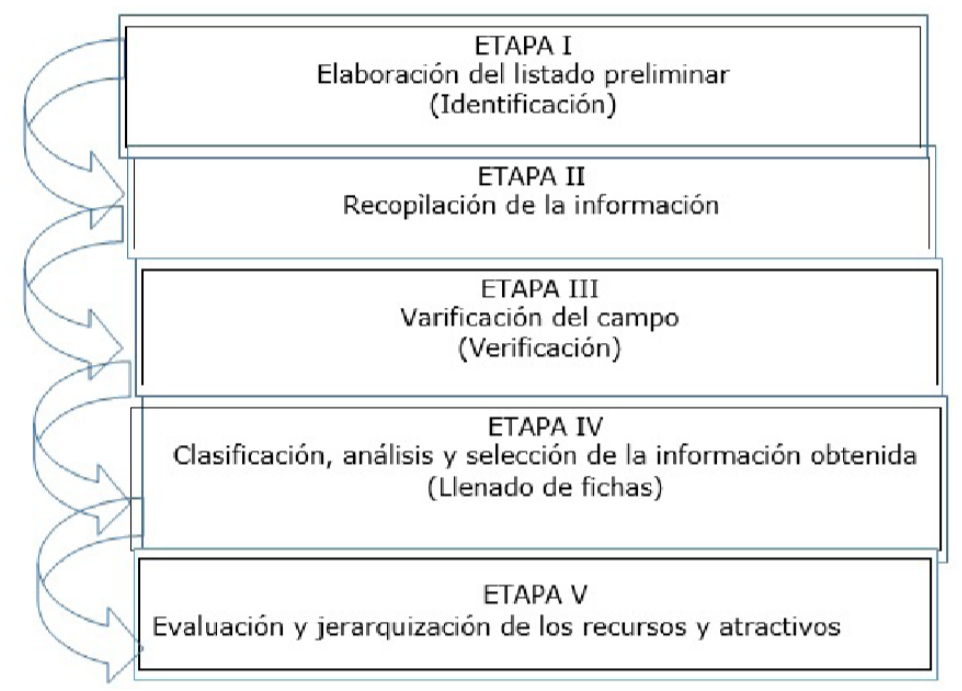

Fuente 8 Ministerio de Culturas y Turismo de Bolivia (2011)

\section{Desarrollo}

En la Etapa 1 se listarán los recursos obtenidos a partir de fuentes bibliográficas en Internet, la Facultad de Geografía, La Universidad de La Habana y la Biblioteca Nacional de Cuba (BNC). Esta etapa permite aproximarnos al contenido y área de estudio y se tendrán en cuenta las clasificaciones, características y detalles de los recursos según la tabla 1.

Tabla 1: Clasificación de los recursos

Categoría: Recursos Básicos Naturales

\begin{tabular}{|c|c|c|}
\hline Tipos & Subtipos & Características \\
\hline Montañas & $\begin{array}{l}\text { Cordillera } \\
\text { Altiplanos } \\
\text { Mesetas, Cerros } \\
\text { Bosque de } \\
\text { Piedras }\end{array}$ & $\begin{array}{l}\text { Altura, morfología, región natural en que } \\
\text { se encuentran. (costa o chala, yunga, etc.) } \\
\text { clima, cambios estacionales, vegetación, } \\
\text { fauna, caminos, senderos y rutas, tipos } \\
\text { de erosión predominante. }\end{array}$ \\
\hline
\end{tabular}




\begin{tabular}{ll}
\hline Planicies & Desiertos \\
Llanuras \\
Tablazos \\
Pampas \\
Salinas \\
Dunas \\
(Médanos)
\end{tabular}

Abras

Cuerpos de agua

Lagos, lagunas

Oasis, pantanos

Albufera,

Humedales

Ríos

Costas

Grutas o Cavernas, Cuevas

Islas, penínsulas Bahías, puntas

Playas

Esteros

Manglares,

caletas
Región natural en que se encuentra (costa o chala, yunga, etc.) clima, cambios estacionales marcados extensión, morfología (permite conocer cómo se han formado la superficie terrestre) vegetación fauna.

Geología clima, altura caminos, senderos, rutas, vegetación, fauna (se asumen a partir de las correspondientes a las quebradas) Región natural en la que se encuentra; extensión, profundidad presencia de islas.

Calidad del agua (color, temperatura, transparencia) flora fauna centros poblados cercanos zonas de pesca, navegables, caudal.

Región natural en la que se encuentra temperatura calidad y propiedades del agua (color, transparencia, salinidad, temperatura) dimensiones y morfología clima, profundidad flora y fauna circundante.

Dimensiones, formación de depósitos: estalactitas, estalagmitas, presencia de cursos de agua internos flora y fauna

Lugar pintoresco de flora o fauna, Bosques, Miradores

Caminos

Pintorescos
Ubicación de sitios extensión, altitud, clima puntos de interés caminos, sendas, rutas especies de interés
Lugares
Pintorescos 


\begin{tabular}{lll}
\hline Creencias & Cuentos & Lugar y fecha de acontecimiento, \\
Populares & Costumbres & $\begin{array}{l}\text { duración. Descripción de la ceremonia, } \\
\text { lugar, tipos de sucesos (histórico, }\end{array}$ \\
& Leyendas & contemporáneo, etc.) \\
& Tradiciones &
\end{tabular}

*De MINCETUR (2006)

Categoría: Recursos Complementarios

\begin{tabular}{ll}
\hline Infraestructura Turística o & Planta turística \\
Infraestructura técnica y de servicios & o Estructura turística
\end{tabular}

\begin{tabular}{|c|c|c|}
\hline Tipo & Subtipo & Tipo \\
\hline Alojamiento & $\begin{array}{l}\text { Hoteles, hostales, } \\
\text { pensiones, casas } \\
\text { para alquilar, } \\
\text { campings, } \\
\text { refugios y } \\
\text { albergues }\end{array}$ & $\begin{array}{l}\text { 1. Redes de transporte } \\
\text { 2. Comunicaciones } \\
\text { 3. Agua y saneamiento } \\
\text { 4. Energía } \\
\text { 5. Salud. } \\
\text { 6. Agua y playa: muelles, carpas, } \\
\text { puentes } \\
\text { 7. Montaña: miradores, senderos }\end{array}$ \\
\hline Restauración & $\begin{array}{l}\text { Restaurantes, } \\
\text { cafeterías y bares }\end{array}$ & \\
\hline $\begin{array}{l}\text { Instalaciones de } \\
\text { ocio }\end{array}$ & $\begin{array}{l}\text { Discotecas, } \\
\text { centros } \\
\text { nocturnos, salas } \\
\text { de fiesta, } \\
\text { piscinas, parques } \\
\text { de diversiones, } \\
\text { centros } \\
\text { deportivos y } \\
\text { pistas } \\
\text { deportivas. }\end{array}$ & \\
\hline $\begin{array}{l}\text { Instalaciones } \\
\text { culturales }\end{array}$ & $\begin{array}{l}\text { Centros } \\
\text { culturales, } \\
\text { galerías de arte, } \\
\text { bibliotecas, cines, } \\
\text { teatros, museos, } \\
\text { asociaciones } \\
\text { culturales. * }\end{array}$ & \\
\hline
\end{tabular}


* Para caracterizarlos se tiene en cuenta ubicación, fecha de fundación, historia, tipo de propiedad, unidades de servicio, estado. Los resultados de las descripciones de este tipo de productos aparecen en la etapa 5

Fuente 1 Elaboración propia a partir de MINCETUR (2006), Jean Camara, Gómez Ortega, \& Morcate Labrada (2019) y Rodríguez (2006)

En la Etapa 2 de Recopilación de información escrita, se realizarán visitas a todas las entidades públicas relacionadas con los mismos, estas son: Instituto de Planificación Física (en la Habana y Mayabeque), la Facultad de Geografía, Biblioteca Nacional de Cuba, Gobierno Provincial (Mayabeque) e Instituto de Geografía Tropical. La información a buscar se refiere a las características o rangos relevantes que le dan interés turístico a los recursos y atractivos identificados en la etapa inicial para presentarla en la ficha de inventario de recursos y atractivos.

En la Etapa 3 Trabajo de Campo, se verifica la validez de la información recopilada en la investigación, para ello se visitarán cada uno de los recursos y atractivos evaluando la vigencia y fidelidad de la información disponible. Se aplicaron entrevistas a especialistas, como a la delegada del Consejo Popular Abras de Canasí y otras entidades en la provincia, con el fin de actualizar o complementar información. También se utiliza como herramienta una guía de observación para identificar nuevos recursos y el estado actual de ellos. Como paso final de esta etapa se muestran consecutivos al listado inicial, los nuevos recursos que fueron identificados. En esta etapa es importante apoyarse en todos los medios disponibles (videos, fotografías, mapas, entro otros).

La Etapa 4 Clasificación, análisis y selección de la información obtenida, consta de dos momentos: el llenado de la Ficha de Inventario y la validación de la metodología empleada que constituye el punto de transición a la etapa 5 .

El inventario de recursos constituye un registro y un estado integrado de todos los elementos turísticos que por sus cualidades naturales, culturales y humanas pueden constituir un recurso para el turista, por lo que representa un instrumento valioso para la planificación turística, toda vez que sirve como punto de partida para realizar evaluaciones y establecer las prioridades necesarias para el desarrollo turístico nacional. (MINCETUR, 2006). 
Para el diseño de la ficha se tomará en cuenta el modelo propuesto por el Viceministerio de Turismo de Perú, al considerarse uno de los más referenciados y completos de los modelos estudiados. No obstante, se modificaron algunos elementos:

- Los aspectos cuyas respuestas coincidían para todos los recursos, se suprimieron de las fichas y se añadieron al inicio del inventario.

- Se suprimieron secciones como la Infraestructura y otros servicios, por considerarse recursos complementarios y unidades de análisis independientes a inventariar.

La validación de la metodología a emplear se realizará a través del método Delphi. Consiste en la utilización sistemática del juicio de un grupo de especialistas para obtener un consenso de opinión. Las principales características del método son: anonimato de los especialistas (ausencia de interrelación), retroalimentación controlada por el facilitador, respuesta estadística de grupo.

Para la validación mediante el método Delphi del procedimiento que se ha seleccionado se siguieron los siguientes pasos:

- I Seleccionar el coordinador;

- II Definir la concepción inicial del problema;

- III Elaborar lista de candidatos a especialista;

- IV Calcular el número de especialistas;

- V Determinar el coeficiente de competencia de cada especialista;

- VI Seleccionar los especialistas;

- VII Trabajar con el grupo de especialistas;

- VIII Calcular la concordancia entre los especialistas

En la Etapa 5, Evaluación y jerarquización de los recursos turísticos, se logrará la estructuración y operatividad del instrumental orientado a la evaluación y jerarquización de los recursos, es decir, hacer tangibles, medibles y registrables las variables.

Para la evaluación se adaptaron las herramientas de Jean Camara, Gómez Ortega, \& Morcate Labrada (2019), que las diferencian según sean los recursos básicos o complementarios. El valor de las jerarquías se diferencia para recursos básicos y complementarios. Además, también se obvió la distinción que hacen dentro de los recursos básicos entre reales y potenciales, y los indicadores de evaluación pertinentes a cada uno: "flujo de turistas" y "demanda potencial", respectivamente; estos fueron fusionados en "demanda de visitantes", como muestra la siguiente tabla: 
Tabla 1 Valores para medir los recursos básicos

\begin{tabular}{|c|c|c|c|c|c|}
\hline \multirow{2}{*}{$\begin{array}{l}\text { Indicadores } \\
\text { de evaluación }\end{array}$} & \multicolumn{4}{|c|}{ Criterios de medición del recurso (puntaje) } & \multirow{2}{*}{ Ponderación } \\
\hline & 6 & 4 & 2 & 1 & \\
\hline Singularidad & Único & Raro & Escaso & Normal & 3 \\
\hline $\begin{array}{l}\text { Imagen } \\
\text { turística real }\end{array}$ & Excepcional & $\begin{array}{l}\text { Muy } \\
\text { bueno }\end{array}$ & Bueno & Normal & 2 \\
\hline $\begin{array}{l}\text { Estado de } \\
\text { conservación }\end{array}$ & Muy bueno & Bueno & Regular & Malo & 1,5 \\
\hline $\begin{array}{l}\text { Demanda } \\
\text { turística }\end{array}$ & Internacional & Nacional & Municipal & Local & 1 \\
\hline
\end{tabular}

Fuente 2 Elaboración propia basada en Jean Camara, Gómez Ortega, y Morcate Labrada (2019)

Los datos que proponen para la evaluación en su ficha de jerarquización para los recursos básicos se adjuntarán al modelo de ficha de inventario de Mincetur (2009) definido en la etapa 4. Los indicadores estado de conservación y demanda turística se sustituyen por "coherencia con los recursos naturales" y "conocimiento del recurso", con el objetivo de saber su popularidad y facilidad para recrearlos en el entorno, como a continuación se expone:

Tabla 2 Ficha de jerarquización para recursos básicos.

\begin{tabular}{|c|c|c|c|c|}
\hline \multicolumn{2}{|c|}{ Jerarquización } & \multicolumn{3}{|c|}{ Valor } \\
\hline Código & Factores & $\begin{array}{l}\text { Valor } \\
\text { asignado }\end{array}$ & Ponderación & Subtotal \\
\hline$S$ & Singularidad & & 2.5 & \\
\hline IP & $\begin{array}{l}\text { Imagen } \\
\text { potencial }\end{array}$ & & 2 & \\
\hline $\mathrm{EC}$ & $\begin{array}{l}\text { Estado de } \\
\text { Conservación }\end{array}$ & & 1.5 & \\
\hline $\mathrm{DT}$ & $\begin{array}{l}\text { Demanda } \\
\text { turística }\end{array}$ & & 1 & \\
\hline Total & & & & \\
\hline
\end{tabular}

En cuanto a los recursos complementarios, no se tuvo en cuenta la clasificación que estos autores dan de los recursos superestructurales al no ser concebidos como parte de los recursos turísticos como análisis de la autora sino como elementos de gestión turística. Por tanto, hubo que reajustar dichos instrumentales, eliminando simplemente todas las variables que ellos consideran para los mismos. Para evaluarlos se procedió con la aplicación del cuestionario, y toma de los indicadores y valores que los autores proponen. 
Los actores que evaluaron dichos recursos fueron un miembro del DPPF Mayabeque, la delegada del Consejo Popular Canasí Mercedes Enseñat y el delegado de la Circunscripción de Boca de Canasí Robert Pierre.

\section{Resultados de la investigación}

Al organizar los resultados de la investigación en esta sección podrá encontrar primero el procedimiento para validar el proceso metodológico; el sistema de indicadores y colores para evaluar y jerarquizar los recursos que de aquel se definen; las fichas de inventarios de los recursos básicos con sus particulares jerarquizaciones; y finalmente un resumen de las jerarquías de este tipo de recursos y la pertinente a los complementarios.

\section{Etapa 4: clasificación, análisis y selección de la información obtenida Validación del procedimiento metodológico}

\section{Paso I: Seleccionar la coordinadora}

El coordinador del proceso de validación del procedimiento mediante el método Delphi es la autora de la investigación.

\section{Paso II: Definir la concepción inicial del problema.}

Se hace necesario demostrar la viabilidad del procedimiento definido entre alrededor de los revisados para la determinación del más adecuado a las condiciones y recursos de Canasí.

Paso III: Elaborar lista de candidatos a especialistas. Para la elaboración de la lista de candidatos a especialistas se toma en consideración la necesidad de contar con un equipo multidisciplinario que analice desde los puntos de vista del turismo y la geografía; para ello se analizan los siguientes requisitos: Motivación e interés en el tema, años de experiencia en la actividad de la salud, conocimiento sobre gestión del turismo de naturaleza. Luego de realizar los análisis pertinentes, los candidatos a especialistas que se seleccionan totalizan 11, de ellos 4 especialistas en geografía; 4 especialistas en turismo, y 3 en ordenamiento territorial.

\section{Fichas de inventario}

A continuación, se muestra la leyenda consensuada a utilizar por el grupo de especialistas para evaluar los recursos (Tabla 7) luego de analizar la información de las fichas que la suceden. 
Tabla 7 Leyenda para la jerarquización a tabular

\begin{tabular}{llll}
\hline LEYENDA & Jerarquía & Posible puntuación & Evaluación \\
\hline & 1 & Menos de 10 puntos & Mal \\
2 & $20-10$ puntos & Regular \\
3 & $30-20$ puntos & Bien \\
4 & $40-30$ puntos & Excelente \\
\hline
\end{tabular}

Fuente 8 Elaboración propia

\section{FICHA No. 1 \\ NOMBRE DEL RECURSO TURISTICO: \\ Río de Canasí}

CATEGORIA: Recurso básico natural TIPO: Río

Descripción:

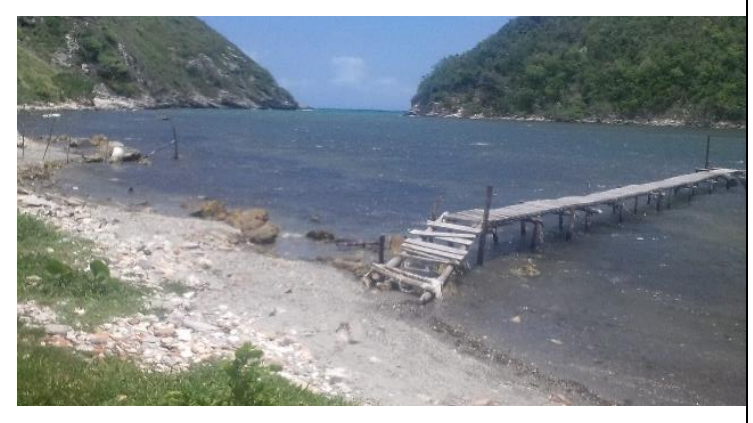

Vertiente Norte. Nace en la Sierra de Los Camarones, alturas de HabanaMayabeque-Matanzas, $7 \mathrm{~km}$ al suroeste del poblado de Arcos de Canasí, $14 \mathrm{~km}$ al este del pueblo de Santa Cruz del Norte, en los $23^{\circ} 02^{\prime} 47^{\prime \prime}$ lat. N y los $81^{\circ} 49^{\prime} 00^{\prime \prime}$ long. O, a 145 m de altitud. Desemboca en la costa norte, en los $23^{\circ} 08^{\prime} 23^{\prime \prime}$ lat. N y los 81 $46^{\prime} 44^{\prime \prime}$ long. O, municipio de Santa Cruz del Norte, Mayabeque. Largo 19,1 km; ancho promedio 150 metros. Área de la cuenca de Canasí 87, 0 km2. Profundidad máxima: 3,6 m. Corre en dirección SN y tiene 4 afluentes. Sus aguas corren por una zona de pendientes abrupta. La vegetación está compuesta fundamentalmente, por macío, malanga de río y manigua.

\section{ESTADO ACTUAL:}

El río se encuentra en un óptimo estado en cuestiones limpieza.

\section{OBSERVACIONES:}

A partir del área otorgada para el desarrollo del producto es válido destacar que no se incluye en la misma el río completo desde su origen, sino solo una parte de él, desde la Vía Blanca hasta su desembocadura

TIPO DE VISITANTE:

1 Extranjero 2 Nacional 2 Regional 3 Local

\section{INFRAESTRUCTURA}

Desagüe

\section{ACTIVIDADES DESARROLLADAS DENTRO DE RECURSO TURÏSTICO} Observación de aves, Pesca de altura, Paseos en bote, Estudios e Investigación Toma de fotografías y filmaciones 


\begin{tabular}{|l|l|l|l|l|}
\hline \hline Jerarquización & Valor asignado & Ponderación & 3 \\
\hline & Factores & 4 & 3 & 12 \\
\hline S & Singularidad & 2 & 2 & 4 \\
\hline IT & Imagen turística potencial & 4 & 1.5 & 6 \\
\hline EC & Estado de conservación & 1 & 1 & 1 \\
\hline DP & Demanda potencial & 1 & 23 \\
\hline \multicolumn{2}{l}{ Total }
\end{tabular}

FICHA No. 2

Llanura ondulada

CATEGORIA: Recurso

básico natural

TIPO: Lugar Pintoresco

SUBTIPO:

Lugar

Pintoresco de Flora.

Descripción:

Se encuentra al suroeste de la Ensenada de Canasí, al sur de las elevaciones del este del abra. Esta área está ocupada actualmente por parte del asentamiento rural. $\mathrm{Su}$ área es de $31,90 \mathrm{~km} 2$. Los suelos son medianamente profundos $(51-90 \mathrm{~cm})$.

ESTADO ACTUAL:

Grado de erosión medio

\section{OBSERVACIONES:}

Boca de Canasí: circunscripción \#17 del Consejo Popular Canasí. Tiene una población de 684 habitantes y 230 unidades de alojamiento.

TIPO DE VISITANTE:

1 Extranjero 2 Nacional 2 Regional 3 Local

\begin{tabular}{|l|l|c|c|c|}
\hline \multicolumn{2}{|l|}{ Jerarquización } & 1 \\
\hline & Factores & Valor asignado & Ponderación & Subtotal \\
\hline S & Singularidad & 1 & 3 & 3 \\
\hline IT & Imagen turística potencial & 2 & 2 & 4 \\
\hline EC & Estado de conservación & 4 & 1.5 & 6 \\
\hline DP & Demanda potencial & 2 & 1 & 2 \\
\hline Total & & 15 \\
\hline
\end{tabular}




\section{FICHA No.4}

\section{Boca de Canasí}

CATEGORIA: Recurso básico natural TIPO: Cuerpo de agua SUBTIPO: Boca

Descripción:

Costa norte de la isla de Cuba, $14,7 \mathrm{~km}$ al E del pueblo de Santa Cruz del Norte, en los $23^{\circ} 08^{\prime} 40^{\prime \prime}$ lat. $\mathrm{N}$ y los $81^{\circ} 46^{\prime} 48^{\prime \prime}$ long. O. Ancho:

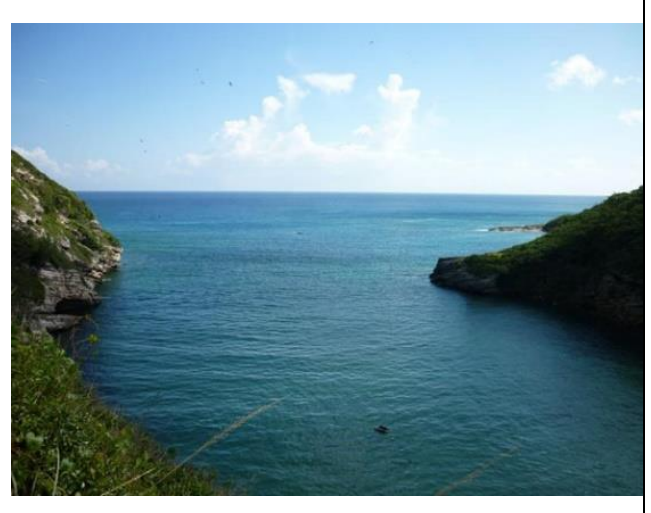

150 m. Profundidad máxima: 3,6 m da entrada al río Canasí. Las puntas de su entrada son relativamente altas, y en la desembocadura del río se ha formado una barra de arena de $0,9 \mathrm{~m}$ de altura.

\section{ESTADO ACTUAL:}

Fuerte grado de erosión. Lugar virgen.

\section{OBSERVACIONES:}

Las costas y la barrera de arena impiden que en mal tiempo la ensenada se vea afectada por el mismo.

\section{TIPO DE VISITANTE:}

1 Extranjero 2 Nacional 2 Regional 3 Local

\section{ACTIVIDADES DESARROLLADAS DENTRO DE RECURSO TURÏSTICO}

Pesca de altura, Paseos en bote, Buceo, Natación, Estudios e Investigación, Toma de fotografías y filmaciones.

\begin{tabular}{|c|c|c|c|}
\hline \multicolumn{3}{|l|}{ Jerarquización } & 4 \\
\hline Factores & Valor asignado & Ponderación & Subtotal \\
\hline Singularidad & 4 & 3 & 12 \\
\hline Imagen turística potencial & 6 & 2 & 12 \\
\hline Estado de conservación & 6 & 1.5 & 9 \\
\hline Demanda potencial & 2 & 1 & 2 \\
\hline \multicolumn{3}{|l|}{ Total } & 35 \\
\hline
\end{tabular}




\begin{tabular}{|c|c|c|c|c|}
\hline \multicolumn{5}{|c|}{$\begin{array}{l}\text { FICHA No. } 5 \\
\text { Arcos de Canasí } \\
\text { CATEGORIA: Recurso básico natural } \\
\text { TIPO: Sierra }\end{array}$} \\
\hline \multicolumn{5}{|c|}{$\begin{array}{l}81^{\circ} 45^{\prime} 00^{\prime \prime} \text { long. Altura máxima: } 123 \mathrm{~m} \text {. Se } \\
\text { presenta en forma de colinas y alturas abrasivo - cársicas: escalonadas en forma de mesa, } \\
\text { sobre calizas con material xerofilico espinoso con suculentos sobre renazina roja poco } \\
\text { profunda y afloramiento de roca. Está cubierta por un monte y manigua espeso con gran } \\
\text { diversidad de especies Presenta en su interior una cueva notable por sus dimensiones. } \\
\text { Antigua, zona de palenques en la colonia. }\end{array}$} \\
\hline \\
\hline \multicolumn{5}{|c|}{1 Extranjero 3 Nacional 2 Regional 2 Local } \\
\hline \multicolumn{5}{|c|}{$\begin{array}{l}\text { ACTIVIDADES DESARROLLADAS DENTRO DE RECURSO TURÏSTICO } \\
\text { Observación de aves, Observación de fauna, Observación de flora, Caminata, } \\
\text { Camping, Excursiones, Escalada en roca, Parapente, Estudios e Investigación, Toma de } \\
\text { fotografías y filmaciones }\end{array}$} \\
\hline \multicolumn{4}{|c|}{ Jerarquización } & \\
\hline & \multirow{2}{*}{$\begin{array}{l}\text { Valor asignado } \\
6\end{array}$} & \multicolumn{2}{|l|}{ Ponderación } \\
\hline $\mathrm{S}$ & Singularidad & & 3 & 18 \\
\hline IT & Imagen turística potencial & 6 & 2 & 12 \\
\hline EC & Estado de conservación & 3 & 1.5 & 4.5 \\
\hline $\mathrm{DP}$ & Demanda potencial & 5 & 1 & 5 \\
\hline \multicolumn{4}{|c|}{ Total } & 39.5 \\
\hline
\end{tabular}




\section{FICHA No. 6 \\ La Cazuela}

CATEGORIA: Recurso básico natural TIPO: Gruta

Descripción:

Está ubicado $3 \mathrm{~km}$ al este del río. Es un conjunto de pequeñas grutas, tres en total,

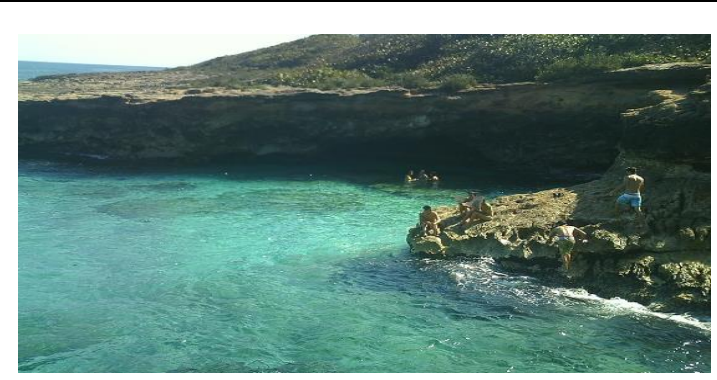
formadas por la acción del mar sobre la costa característica de la zona en forma quebradiza. Es una especie de lecho pedregoso. Presenta cardúmenes de agujitas.

$\mathrm{Al}$ adentrarse en la cueva principal la profundidad va descendiendo, y cruzado el ancho umbral recibe a los curiosos una explanada de arena muy fina. Un verdadero descanso para los pies cansados de tanto caminar.

ESTADO ACTUAL:

Virgen.

TIPO DE VISITANTE:

1 Extranjero 3 Nacional 2 Regional 2 Local

ACTIVIDADES DESARROLLADAS DENTRO DE RECURSO TURISTICO

Natación y Buceo

\begin{tabular}{|l|l|l|l|l|}
\hline \multicolumn{2}{|c|}{ Jerarquización } & Valor asignado & Ponderación & Subtotal \\
\hline & Factores & 6 & 3 & 18 \\
\hline S & Singularidad & 2 & 12 \\
\hline IT & Imagen turística potencial & 6 & 1.5 & 6 \\
\hline EC & Estado de conservación & 4 & 1 & 5 \\
\hline DP & Demanda potencial & 5 & 41 \\
\hline \multicolumn{2}{|l|}{ Total }
\end{tabular}




\section{FICHA No. 7 \\ Playitas de Canasí}

CATEGORIA: Recurso básico natural TIPO: Costa

SUBTIPO: Playas

\section{Descripción:}

La primera zona de playa es muy pequeña, está ubicada a unos 100 metros al este de La cazuela. Es

de corta extensión, apenas, unos 50 metros. Existe como consecuencia de la acción del mar sobre litoral costero de la zona rocoso, por lo que se encuentra limitada por columnas rocosas. La otra zona es más extensa y supera los límites de área de estudio. Ambas zonas poseen aguas cristalinas y cálidas. Poseen arena fina en su orilla, pero al penetrar en el mar se notan formaciones rocosas y pequeños arrecifes. Son comunes los cangrejos.

ESTADO ACTUAL:

Virgen.

TIPO DE VISITANTE:

1 Extranjero 3 Nacional 2 Regional 2 Local

ACTIVIDADES DESARROLLADAS DENTRO DE RECURSO TURÏSTICO Natación y Buceo

\begin{tabular}{|c|c|c|c|}
\hline \multicolumn{3}{|l|}{ Jerarquización } & 3 \\
\hline Factores & Valor asignado & Ponderación & Subtotal \\
\hline Singularidad & 4 & 3 & 12 \\
\hline Imagen turística potencial & 4 & 2 & 8 \\
\hline Estado de conservación & 4 & 1.5 & 6 \\
\hline Demanda potencial & 5 & 1 & 5 \\
\hline \multicolumn{3}{|l|}{ Total } & 31 \\
\hline
\end{tabular}




\section{FICHA No. 8 Historia de Canasí}

CATEGORIA: Recurso básico cultural

TIPO: Creencia popular

SUBTIPO: Leyenda

Descripción:

Es una leyenda aborigen de la cual toman nombre muchos accidentes geográficos del municipio de Santa Cruz del NorteCuentan que Bamayagua, madre de Canasí, gobernaba sabiamente su aldea, la cual aspiraba que su hijo fuera cacique de todo el valle, por lo que pretendía casarlo con Albahoa, hija del cacique Guananey, pero este había prometido la mano de su hija al cacique Yumurí, indio yucayo. Sufría Bamayagua por este compromiso y trataba por todos los medios de romperlo.

Camují, guerrero de confianza del cacique Guananey amaba a la joven viuda Bamayagua, la que se vale de sus amores, le ofrece su mano en matrimonio, si él consigue la ruptura del noviazgo de Albahoa y Yumurí.

Tiempo después en la bahía de Yucayo surge una disputa entre las tribus donde muere un guerrero del cacique Guananey, el cacique instigado por Camují y Bamayagua rompe toda relación con Yumurí, hasta el noviazgo de su hija.

Lunas después se celebran bodas: Camují - Bamayagua, Canasí- Albahoa.

Yumurí y Albahoa se amaban, el cacique yucayo estaba atento y había trazado un plan de fuga.

Ambas aldeas disfrutaban los festejos, pero Albahoa esperaba la señal de su amado, al escucharla sale con sigilo, pero es vista por un guerrero que da la alarma. Corren Albahoa y Yumurí tomados de las manos, la joven tropieza y se lastima, el guerrero la carga, corren, los persiguen.

Yumurí comprende que es imposible llegar a las canoas, deben cruzar el río y toma la parte del valle por donde el río es más estrecho y forma una garganta de piedra, era una zona cenagosa, salta sobre los mangles, crujen bajo el peso de los cuerpos, pisa el fango, este se los va tragando, ante los ojos asombrados de sus perseguidores.

Lunas después queda como cacique en todas estas tierras Canasí.

\begin{tabular}{|c|c|c|c|c|}
\hline \multicolumn{4}{|c|}{ Jerarquización } & 3 \\
\hline & Factores & Valor asignado & Ponderación & Subtotal \\
\hline $\mathrm{S}$ & Singularidad & 6 & 3 & 18 \\
\hline IP & Imagen turística potencial & 2 & 2 & 4 \\
\hline EC & Coherencia con el medio & 5 & 1.5 & 7.5 \\
\hline DP & Conocimiento local & 1 & 1 & 1 \\
\hline \multicolumn{4}{|c|}{ Total } & 30.5 \\
\hline
\end{tabular}

\section{Etapa 5: jerarquización y evaluación de los recursos}

Por otro lado, las facilidades que existen para el disfrute del atractivo dentro del área otorgada satisfacen la demanda actual que posee el sitio como lugar de acampada, pero no para el desarrollo de un parque temático. Lo más parecido a ello es el terraplén que facilita la llegada al abra desde la Vía Blanca y una casa de renta llamada Monte Corales. De hecho, los recursos complementarios, específicamente la planta turística es muy débil. Fuera del área otorgada, a unos 5km se encuentran los hoteles Villa Trópico y Memories 
Jibacoa y los Campismos Peñas Blancas y Playa Amarilla, que podrían considerarse instalaciones que complementen el producto principal con servicio de alojamiento, o bien dirigir el producto parque temático a un segmento de demanda objetivo con un perfil semejante al de dichas instalaciones para diversificar las ofertas opcionales del mismo ya que dichas entidades, al poseer jerarquía 3, en combinación con los recursos naturales contiguos llegan a generar flujo de visitantes importante.

Se considera que los recursos complementarios infraestructurales son de gran valor para el desarrollo del producto en cuestión. Dentro del área, la población se abastece diariamente de agua y energía eléctrica. Pero los existentes afuera presentan jerarquía 2, como el transporte. Es importante valorar además la cercanía del producto con zonas petrolíferas y de gas en desarrollo, lo cual representan también posible fuente de energía.

Tabla 4 Jerarquización de recursos básicos

\begin{tabular}{llll}
\hline $\begin{array}{l}\text { Jerarquía 1: } \\
\text { Mal }\end{array}$ & $\begin{array}{l}\text { Jerarquía 2: } \\
\text { Regular }\end{array}$ & $\begin{array}{l}\text { Jerarquía } \\
\text { 3: Bien }\end{array}$ & $\begin{array}{l}\text { Jerarquía 4: } \\
\text { Excelente }\end{array}$ \\
\hline & & Río Canasí & $\begin{array}{l}\text { Arcos de } \\
\text { Canasí, La }\end{array}$ \\
& & Cazuela, \\
& Llanura & & Ensenada de \\
ondulada & Playa, & Canasí \\
& & Leyenda & \\
\hline
\end{tabular}

Fuente 5 Elaboración propia

Tabla 5 Evaluación de los recursos complementarios (planta turística)

\begin{tabular}{|c|c|c|c|}
\hline Tipos & Unidades & Unidades & Tipo \\
\hline \multirow{3}{*}{ 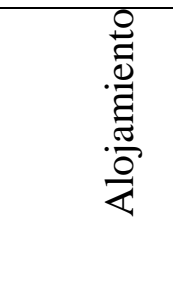 } & Hoteles & 5 & \\
\hline & $\begin{array}{l}\text { Casa para } \\
\text { alquilar }\end{array}$ & 3 & \\
\hline & Campismos & 4 & \\
\hline \multirow{6}{*}{ 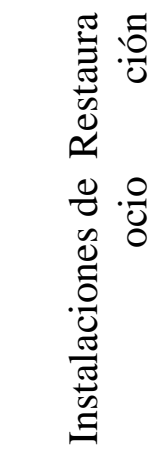 } & Cafeterías & 2 & \\
\hline & Bares & 2 & \\
\hline & Restaurante & 3 & \\
\hline & $\begin{array}{l}\text { Centros } \\
\text { nocturnos }\end{array}$ & \multirow{3}{*}{2.99} & \\
\hline & $\begin{array}{l}\text { Casa de la } \\
\text { música }\end{array}$ & & \\
\hline & Salas de fiestas & & \\
\hline
\end{tabular}




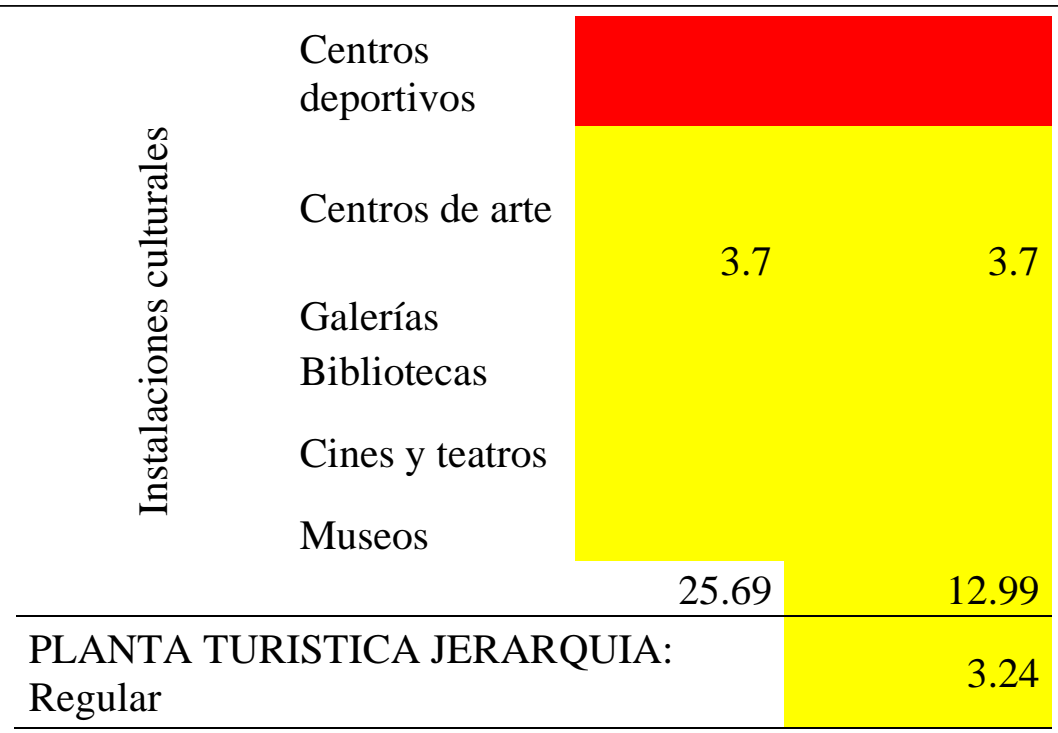

Fuente 6 Elaboración propia

Tabla 6 Jerarquización de recursos complementarios infraestructurales.

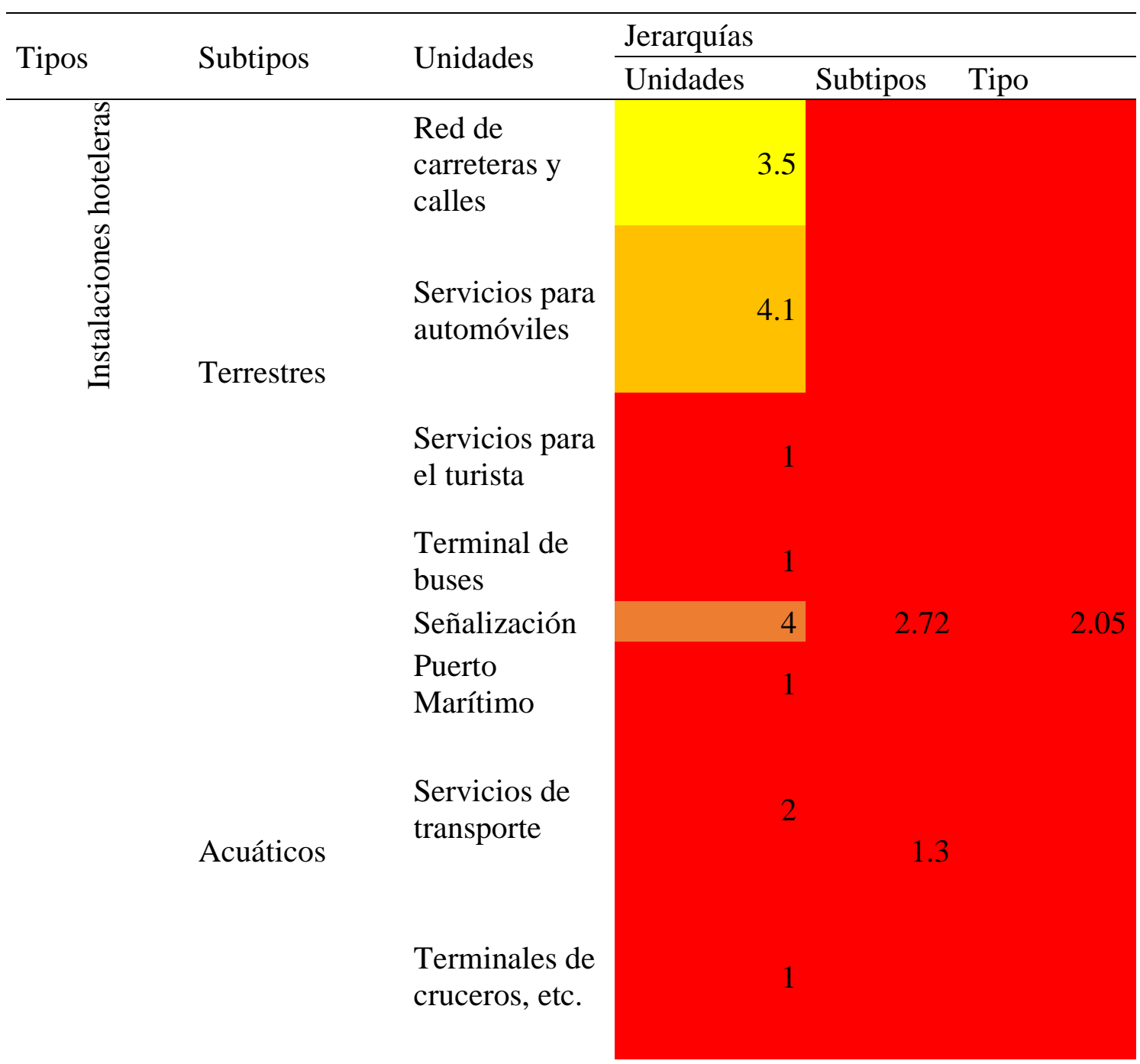


Servicios

Oficinas de correos

estáticos (fijos)

Centrales telefónicas

Servicios de Telefonía redes

Internet Acueducto (agua potable)
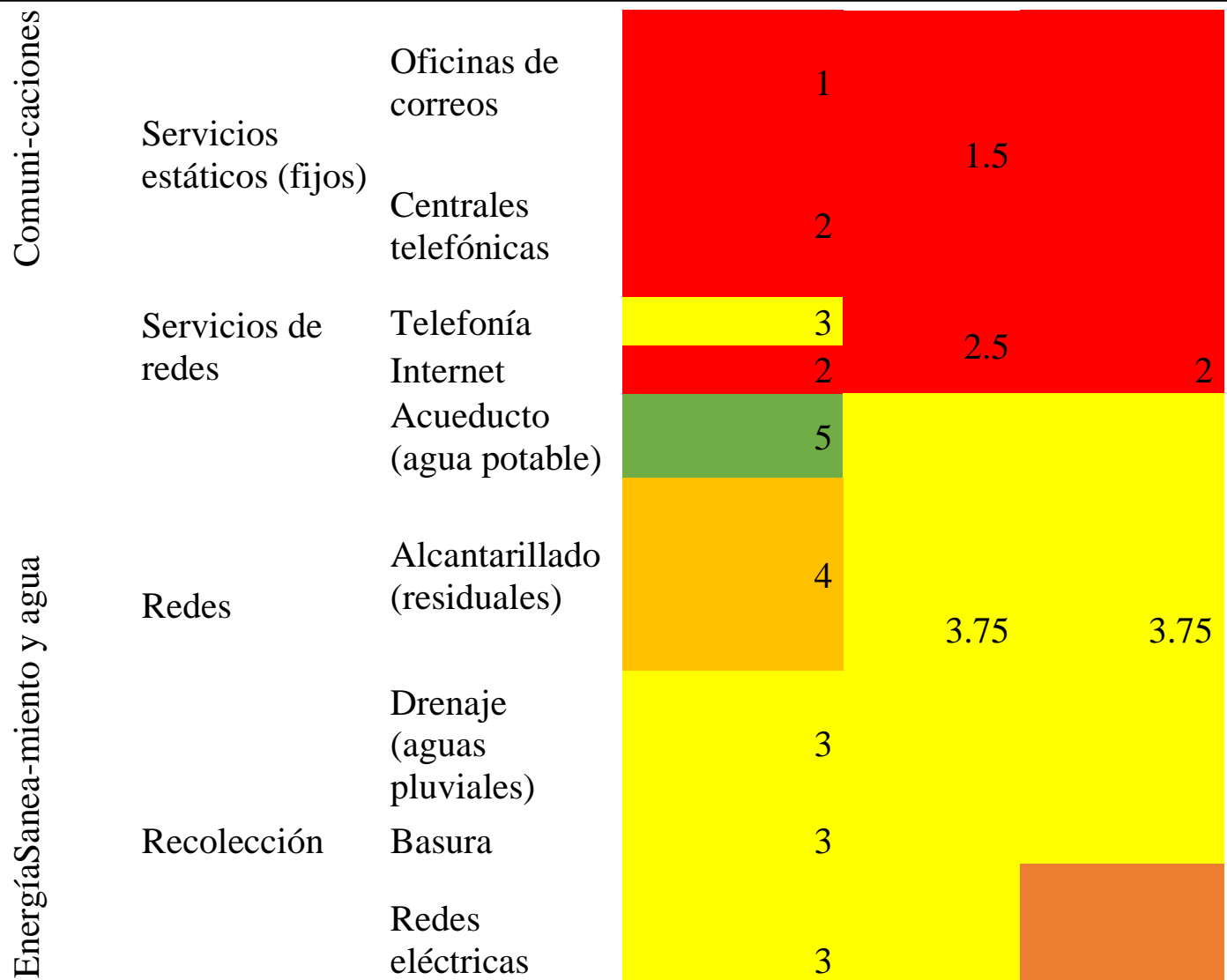

Alcantarillado (residuales)

4

Drenaje

(aguas pluviales)

Redes

Basura

Redes

eléctricas

principales

3

Redes

eléctricas

públicas

Combustibles Gasolina

4

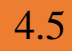

Gas

5

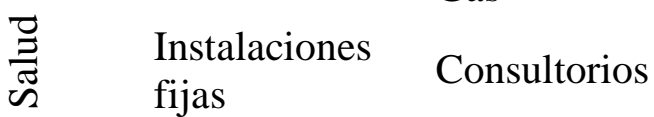

3

Ambulatorios Urgencia 3

\begin{tabular}{lrrr}
\hline TOTAL & 61.6 & 22.7 & 14.55 \\
\hline $\begin{array}{l}\text { Recursos complementarios infraestructurales } \\
\text { (JERARQUIA 1) }\end{array}$ & & & 2.9 \\
\hline
\end{tabular}

Fuente 7 Elaboración propia

\section{Conclusiones}

- La zona de Canasí es un área propicia para el desarrollo de modalidades como el turismo de naturaleza, los parques temáticos, el ecoturismo y turismo de aventuras. Np obstante la leyenda local le otorga un valor cultural atractivo para 
actividades de este tipo y la posibilidad de asociar el concepto de productos como parques temáticos a tal recurso. También se puede valorar el desarrollo de productos de ocio que complementen la oferta hotelera presente en la zona y analizar la sostenibilidad del medio para preservar el medio ambiente.

- El resultado de un análisis exhaustivo mediante tablas, gráficos y valoraciones de las diversas metodologías y procedimientos encontrados, permite determinar a la metodología para la inventariación, categorización y jerarquización de los recursos y atractivos turísticos del colectivo de autores Charles Jean Camara, Graciela Gómez Ortega y Flora Morcate Labrada (2019), como la más indicada y la que más se adapta a la investigación, y para la clasificación de los recursos turísticos, la del Viceministro de Bolivia (2011).

- El inventario definido constituye una herramienta que otorga a los profesionales un medio para evaluar el potencial de otros productos turísticos territoriales o espacios turísticos, así como comercializar espacios que aún no poseen valor turístico.

\section{Referencias bibliográficas}

Centro Nacional de Areas Protegidas. (2009). Planilla expediente Boca de Canasí. La Habana: CNAP.

Colina, J. (2009). Marketing turístico. Buenos Aires, Argentina: El Cid Editor.

DPPF Mayabeque. (2018). Santa Cruz Actualización de Asentamientos Humanos. San José de las Lajas: Dirección de Planificación Física de Mayabeque.

Ecured. (19 de octubre de 2018). Canasí (Santa Cruz del Norte). Obtenido de Canasí (Santa Cruz del Norte): «https://www.ecured.cu/index.php?title=Canasí_(Santa_Cruz_del_Norte)\&oldid $=2453614 »$

Fayos-Solá, E., Muñoz, A., \& Fuentes, L. (2011). El modelo FAS. Orígenes, Desarrollo y Aplicación en Destinos Turísticos. (188). Estudios Turísticos.

Font Aranda, M. (2013). Inventario de atractivos para el desarrollo turístico local del municipio Martí. Revista Avanzada Científica., 16(2). Obtenido de https://dialnet.unirioja.es/descarga/articulo/4325393. pdf

Gavín Morales, D. (2019). Evaluación de los recursos y atractivos para el desarrollo del turismo de salud en Cienfuegos. Universidad y Sociedad, 11(3), 301-314. Obtenido de http://rus.ucf.edu.cu/index.php/rus

González, P. (2016). Diseño de Productos y Servicios Turísticos Locales. La Rioja: Tutor formación.

Izquierdo Ferrer, L. (30 de noviembre de 2017). Promueven proyectos para desarrollar centros recreativos en Cuba. Obtenido de http://www.acn.cu 
Jean Camara, C., Gómez Ortega, G., \& Morcate Labrada, F. (enero-abril de 2019). Instrumental para evaluar los recursos territoriales turísticos del centro de ciudad de Fort-de -Franc. Con Criterio, XV(1), 52-71.

MINCETUR. (2006). Manual para la formulación del inventario de recursos turísticos a nivel nacional. Viceministerio de Turismo, Dirección Nacional de Desarrollo Turístico.

Ministerio de Comercio Exterior y de la Inversión Extranjera. (2017). Cartera de oportunidades de Inversión Extranjera 2017-2018. Obtenido de Cartera de oportunidades de Inversión Extranjera 2017-2018: http://www.camaracuba.cu

Ministerio de Culturas y Turismo. (2011). Metodología para la inventariación, categorización y jerarquización de los recursos y atractivos turísticos. Ministerio de Culturas y Turismo, La Paz. Bolivia.

Navarro, D. (1 de 1 de 2015). Recursos turísticos y atractivos turísticos: conceptualización, clasificación y valoración. Cuadernos de Turismo(35), 335357. doi:https://doi.org/10.6018/turismo.35.221641

OMT. (1978). Evaluación de los recursos turísticos. Madrid: Organización Mundial del Turismo.

OMT. (15 de enero de 2018). Resultados del turismo internacional en 2017: los más altos en siete años. Obtenido de Resultados del turismo internacional en 2017: los más altos en siete años: http://media.unwto.org/es/press-release/2018-0115/resultados-del-turismo-internacional-en-2017-los-mas-altos-en-siete-anos

Organización Mundial del Turismo . (2018). ¿Por qué el turismo? Organización Mundial del Turismo OMT. Obtenido de http://www2.unwto.org/es/content/por-que-elturismo

Partido Comunista de Cuba. (2017). Lineamientos de la Política Económica y Social del Partido y la Revolución para el período 2016-2021. La Habana.

Sierra López, A. (2006). Recursos turísticos: inventario, clasificación, jerarquización y evaluación.

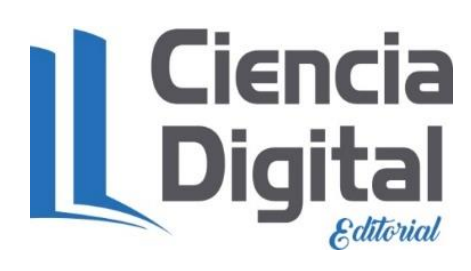




\section{PARA CITAR EL ARTÍCULO INDEXADO.}

Morales Blanco, A. (2021). Evaluación de los recursos turísticos en Canasí. Explorador Digital, 5(1), 81-102. https://doi.org/10.33262/exploradordigital.v5i1.1491

\section{Ciencia \\ LDigital}

El artículo que se publica es de exclusiva responsabilidad de los autores y no necesariamente reflejan el pensamiento de la Revista Explorador Digital.

El artículo queda en propiedad de la revista y, por tanto, su publicación parcial y/o total en otro medio tiene que ser autorizado por el director de la Revista Explorador Digital.
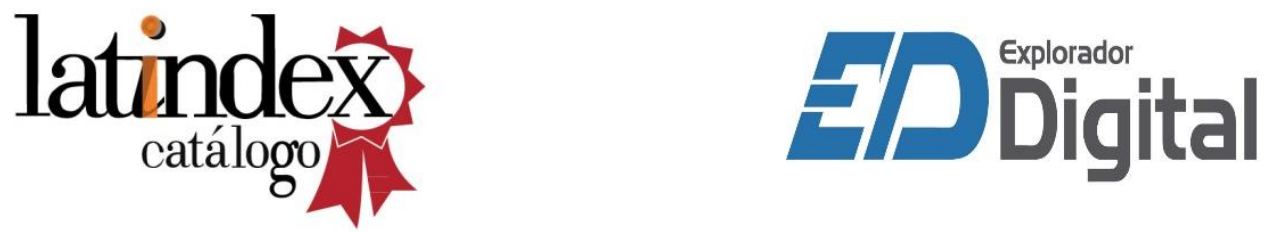